\title{
A CONSTRUCTIVE SOLUTION FOR A GENERALIZED THOMAS-FERMI THEORY OF IONIZED ATOMS*
}

\author{
BY \\ C. Y. CHAN AND Y. C. HON \\ University of Southwestern Louisiana
}

1. Introduction. Thomas [13] and Fermi [5] independently studied the nonlinear secondorder differential equation

$$
y^{\prime \prime}=x^{-1 / 2} y^{3 / 2}
$$

when they were working on potentials and charge densities in atoms. The boundary conditions in investigating

(a) the neutral atom with Bohr radius $b$ are given by

$$
y(0)=1, \quad b y^{\prime}(b)-y(b)=0
$$

(b) the isolated neutral atom are given by

$$
y(0)=1, \quad \lim _{x \rightarrow \infty} y(x)=0 ;
$$

(c) the ionized atom are given by

$$
y(0)=1, \quad y(a)=0 .
$$

The boundary value problem (1.1) and (1.2) was studied by Luning [7], who showed that for certain values of $b$, a sequence of functions obtained from the solutions of a related linear eigenvalue problem converges uniformly on the interval $[0, b]$ to the solution. Approximate solutions of the problem (1.1) and (1.3) were given, for example, by Bush and Caldwell [3], Sommerfeld [12], Ramnath [10], and more recently by Anderson and Arthurs [1], and Burrows and Core [2] using the variational approach. The case of the ionized atom given by (1.1) subject to (1.4) was studied by Mooney [8] using monotone methods with both (modified) Picard and Newton algorithms, and recently by Chan and $\mathrm{Du}[4]$.

In this paper, we generalize (1.1) to the form

$$
y^{\prime \prime}+(b / x) y^{\prime}=c x^{p} y^{q}
$$

\footnotetext{
${ }^{*}$ Received October 29, 1986.
} 
where $b, c, p$, and $q$ are constants such that $0 \leqslant b<1, c>0, p>-2$, and $q>1$. We study it under the boundary condition (1.4). In Sec. 2, we use modified Bessel functions of the first kind and the second kind to construct Green's function of a linear problem, which is used in each successive approximation to the problem (1.5) and (1.4). In contrast to using modified Bessel functions of the first kind in the paper of Chan and Du, the use of the modified Bessel function of the second kind here enables us to consider also the case when its order is an integer. In Sec. 3, we construct a sequence of lower bounds as well as a sequence of upper bounds, and prove that each sequence converges to obtain existence of a nonnegative solution. Although we use the monotone method, our proof is self-contained, and is different from that of Mooney. Based on the Lebesgue Convergence Theorem (cf. Royden [11, p. 88]), it is due to Keller and Cohen [6] in their study of a nonlinear eigenvalue problem. Uniqueness then follows from the maximum principle (cf. Protter and Weinberger [9, p. 6]). Finally, we establish the dependence of the solution on the size of the interval $[0, a]$; this gives a generalization of a result of Mooney, and a different proof. For illustrations, we apply, in Sec. 4 , the above constructive method to the problem (1.5) and (1.4) with $b=\frac{1}{4}$ and $b=0$, respectively, for $a=1, c=1, p=-\frac{1}{2}$, and $q=\frac{3}{2}$. For each problem, we use the computer to compute the successive lower bounds as well as the successive upper bounds, and show that they converge to the same numerical solution. The case $b=0$ deals with the Thomas-Fermi equation, for which Mooney stated that there were no known analytic solutions (cf. problems (6.1) and (6.3) of his paper) to the iterates. Here, we derive new analytic solutions to all iterates for the more general problem (1.5) and (1.4).

2. Nonhomogeneous linear problem. In this section, we use modified Bessel functions of the first and second kinds to construct Green's function $G(x ; \xi)$, which gives a representation formula for the solution of the following nonhomogeneous problem:

$$
L v=f(x), \quad v(0)=0=v(a),
$$

where $L v$ denotes

$$
\left(x^{h} v^{\prime}\right)^{\prime}-c q x^{p+h} v
$$

With

$$
\begin{aligned}
\alpha & =2(c q)^{1 / 2} i /(p+2), \\
\beta & =(p+2) / 2 \\
\nu & =(1-b) /(p+2),
\end{aligned}
$$

the homogeneous equation,

$$
L w=0
$$

can be written as

$$
x^{b}\left[w^{\prime \prime}+\frac{1-2 \beta \nu}{x} w^{\prime}+\alpha^{2} \beta^{2} x^{2(\beta-1)} w\right]=0,
$$

whose general solution (cf. Watson [14, p. 97]) is given by

$$
w(x)=k_{1} w_{1}(x)+k_{2} w_{2}(x) \text {, }
$$


where $k_{1}$ and $k_{2}$ are arbitrary constants,

$$
w_{1}(x)=x^{(1-h) / 2} I_{\nu}\left(|\alpha| x^{\beta}\right),
$$

with $I_{\nu}$ denoting the modified Bessel function of the first kind of order $\nu$,

$$
w_{2}(x)=x^{(1-b) / 2} K_{\nu}\left(|\alpha| x^{\beta}\right),
$$

with $K_{v}$, denoting the modified Bessel function of the second kind of order $\nu$. Since $b<1$ and $p>-2$, we have $0<v<\infty$. It follows from (2.3) that the homogeneous equation (2.2) subject to $w(0)=0=w(a)$ has the trivial solution only. Hence, the nonhomogeneous problem (2.1) has a unique solution. Using the properties of determinants, and the fact that the Wronskian (cf. Watson [14, p. 80]) of $I_{\nu}(z)$ and $K_{\nu}(z)$ is $-1 / z$, we obtain Green's function,

$$
G(x ; \xi)=\left\{\begin{array}{l}
w_{1}(x)\left[w_{1}(\xi)-k w_{2}(\xi)\right] /(k \beta), 0 \leqslant x \leqslant \xi, \\
w_{1}(\xi)\left[w_{1}(x)-k w_{2}(x)\right] /(k \beta), \xi \leqslant x \leqslant a,
\end{array}\right.
$$

where

$$
k=I_{\nu}\left(|\alpha| a^{\beta}\right) / K_{\nu}\left(|\alpha| a^{\beta}\right)>0 .
$$

Although the maximum principle can be used to show that Green's function is negative, we give a more elementary proof here.

Lemma 1. For $x$ and $\xi$ in the interval $(0, a), G(x ; \xi)<0$.

Proof. To prove the lemma, it is sufficient to show that

$$
I_{\nu}\left(|\alpha| x^{\beta}\right)-k K_{\nu}\left(|\alpha| x^{\beta}\right)<0 \text { for } 0<x<a .
$$

It follows from the value of the Wronskian that

$$
\frac{d}{d x}\left[\frac{I_{\nu}\left(|\alpha| x^{\beta}\right)}{K_{\nu}\left(|\alpha| x^{\beta}\right)}\right]=\frac{\beta}{x}\left[K_{\nu}\left(|\alpha| x^{\beta}\right)\right]^{-2}>0 \text { for } 0<x \leqslant a .
$$

Thus, $I_{\nu}\left(|\alpha| x^{\beta}\right) / K_{\nu}\left(|\alpha| x^{\beta}\right)$ is an increasing function, and we have

$$
I_{\nu}\left(|\alpha| x^{\beta}\right) / K_{\nu}\left(|\alpha| x^{\beta}\right)<k \text { for } 0<x<a,
$$

from which (2.4) follows. Thus, the lemma is proved.

The solution of the nonhomogeneous problem (2.1) is given by

$$
v(x)=\int_{0}^{a} G(x ; \xi) f(\xi) d \xi .
$$

From this and Lemma 1, we have the following positivity result.

LEMMA 2. If $f$ is continuous, nontrivial, and nonpositive, then $v>0$ for $0<x<a$.

3. Existence, uniqueness, and dependence on the interval. If the problem (1.5) and (1.4) has a solution $y$ that is negative somewhere, say at $x_{0}$, in the interval $(0, a)$, and if $q$ is such that $\left[y\left(x_{0}\right)\right]^{q}<0$, then there exists a subinterval $\left(x_{1}, x_{2}\right)$ containing $x_{0}$ such that the right-hand side of (1.5) is negative, and $y\left(x_{1}\right)=0=y\left(x_{2}\right)$. Since the coefficient $b / x$ of $y^{\prime}$ in (1.5) is bounded on every closed subinterval interior to $(0, a)$, we may apply the maximum principle to arrive at a contradiction. Thus, to show that the problem (1.5) and 
(1.4) has a nonnegative solution only, it is sufficient to consider the case where $y^{q} \geqslant 0$ for any solution $y$ since the left-hand side of (1.5) is real.

We note that for any solution $y$ such that $y^{q} \geqslant 0$, if $y$ attains its maximum $M$ somewhere in the interval $(0, a)$, then it follows from the maximum principle that $y \equiv M$ in $(0, a)$, and we have a contradiction. Thus, $y$ cannot attain its maximum inside the interval. Since $y(0)=1$, and $y(a)=0$, it follows from (1.5) with $b \geqslant 0$ and $c>0$ that the graph of $y$ is concave upwards. Therefore, $y \leqslant 1-x / a$ for $0 \leqslant x \leqslant a$. Let

$$
u=1-x / a-y \text {. }
$$

From (1.5) and (1.4), we have, respectively,

$$
\left(x^{h} u^{\prime}\right)^{\prime}=-c x^{p+h}(1-x / a-u)^{q}-(b / a) x^{b-1}, \quad u(0)=0=u(a) .
$$

Let us construct a sequence $\left\{U_{n}\right\}$ as follows:

$$
U_{0}=1-x / a \text { for } 0 \leqslant x \leqslant a ;
$$

for $n=0,1,2, \cdots$,

$$
\begin{gathered}
L U_{n+1}=-x^{p+b}\left[c\left(1-\frac{x}{a}-U_{n}\right)^{q}+c q U_{n}+\frac{b}{a} x^{-(p+1)}\right], \\
U_{n+1}(0)=0=U_{n+1}(a) .
\end{gathered}
$$

To establish an existence result, let us state the following lemma, whose proof follows immediately from the fact that the derivative of the function with respect to $z$ is negative for $q>1$.

Lemma 3. For $0 \leqslant z \leqslant 1-x / a$, where $0<x<a$, the function

$$
-\left[(1-x / a-z)^{q}+q z\right]
$$

is decreasing with respect to $z$.

The following result gives lower bounds and existence of a unique nonnegative solution constructively.

THEOREM 1. The problem (1.5) and (1.4) has a unique solution $y$ ( $>0$ for $0 \leqslant x<a$ ), to which the sequence $\left\{1-x / a-U_{n}\right\}$ converges monotonically upwards.

Proof. Since Green's function $G(x ; \xi)$ corresponding to (3.4) exists, the function $U_{n+1}$ is well defined (provided $0 \leqslant U_{n} \leqslant 1-x / a$ ). From (3.3) and (3.4),

$$
L\left(U_{0}-U_{1}\right)=0, \quad U_{0}(0)-U_{1}(0)=1, \quad U_{0}(a)-U_{1}(a)=0 .
$$

It follows from $(2.2),(2.3)$, and $\Gamma(\nu) \Gamma(1-\nu)=\pi /(\sin \nu \pi)$ that

$$
U_{0}(x)-U_{1}(x)=\frac{2}{\Gamma(\nu)}\left|\frac{\alpha}{2}\right|^{\nu}\left[w_{2}(x)-w_{1}(x) / k\right]
$$

where $\Gamma(\nu)$ is the gamma function, which is positive since $\nu>0$. By (2.4), $U_{0}(x)>U_{1}(x)$ for $0<x<a$. From (3.4) (with $b \geqslant 0, c>0$, and $q>1$ ) and Lemma 2, $U_{1}(x)>0$ for $0<x<a$.

Let us assume that for a particular value of $n$, say $j$,

$$
U_{j-1}>U_{j}>0 \text { for } 0<x<a \text {. }
$$


From (3.4), and Lemma 3,

$$
L\left(U_{j}-U_{j+1}\right)<0 .
$$

It follows from Lemma 2 that for $0<x<a, U_{j}>U_{j+1}$, and $U_{j+1}>0$. By the principle of mathematical induction,

$$
0<U_{n+1}(x)<U_{n}(x) \text { for } 0<x<a, n=0,1,2, \cdots .
$$

Since the sequence $\left\{U_{n}\right\}$ is monotonically decreasing, and is bounded above and below, there exists a function $U(x)$ such that $\lim _{n \rightarrow \infty} U_{n}=U$. To show that $U$ is a solution of (3.2), let us rewrite the iteration scheme (3.4) equivalently as

$$
\begin{aligned}
U_{n+1}(x)=-\int_{0}^{a} G_{0}(x ; \xi) \xi^{p+b}\left\{c\left[1-\frac{\xi}{a}-U_{n}(\xi)\right]^{q}+\frac{b}{a} \xi^{-(p+1)}\right. & \\
& \left.+c q\left[U_{n}(\xi)-U_{n+1}(\xi)\right]\right\} d \xi,
\end{aligned}
$$

where

$$
G_{0}(x ; \xi)=\left\{\begin{array}{l}
x^{1-b}\left(\xi^{1-b}-a^{1-b}\right) /\left[a^{1-b}(1-b)\right], 0 \leqslant x \leqslant \xi, \\
\xi^{1-b}\left(x^{1-b}-a^{1-b}\right) /\left[a^{1-b}(1-b)\right], \xi \leqslant x \leqslant a,
\end{array}\right.
$$

is Green's function corresponding to $\left(x^{b} w^{\prime}\right)^{\prime}=0$ with homogeneous first boundary conditions. The integrand of the above integral is bounded by

$$
-G_{0}(x ; \xi) \xi^{p+b}\left[c\left(1-\frac{\xi}{a}\right)^{q}+\frac{b}{a} \xi^{-(p+1)}+c q U_{0}(\xi)\right],
$$

which is integrable. As $n$ tends to infinity, it follows from the Lebesgue Convergence Theorem that the limit and integration processes can be interchanged so that

$$
U(x)=-\int_{0}^{a} G_{0}(x ; \xi) \xi^{p+b}\left\{c\left[1-\frac{\xi}{a}-U(\xi)\right]^{q}+\frac{b}{a} \xi^{-(p+1)}\right\} d \xi
$$

This implies that $U$ is a solution of the problem (3.2), and hence by (3.1), $y$ is a solution of the problem (1.5) and (1.4). Thus, we have existence of a solution $y$ ( $>0$ for $0 \leqslant x<a)$, to which the sequence $\left\{1-x / a-U_{n}\right\}$ converges monotonically upwards. From the maximum principle, we have exactly one solution.

We can also construct a sequence $\left\{u_{n}\right\}$ as follows:

$$
u_{0} \equiv 0 \text { for } 0 \leqslant x \leqslant a
$$

for $n=0,1,2, \cdots$,

$$
\begin{gathered}
L u_{n+1}=-x^{p+b}\left[c\left(1-\frac{x}{a}-u_{n}\right)^{q}+c q u_{n}+\frac{b}{a} x^{-(p+1)}\right], \\
u_{n+1}(0)=0=u_{n+1}(a) .
\end{gathered}
$$

We state without proof the following result since its proof is similar to that of Theorem 1 . 
THEOREM 2. The problem (1.5) and (1.4) has a unique solution $y$ ( $>0$ for $0 \leqslant x<a$ ), to which the sequence $\left\{1-x / a-u_{n}\right\}$ converges monotonically downwards; furthermore, for $n=0,1,2, \cdots$,

$$
u_{n}<u_{n+1}<U_{0} \text { for } 0<x<a .
$$

Let $x=a t$, and $\zeta(t)=y(a t)$. Then, (1.5) and (1.4) become

$$
\begin{gathered}
\zeta^{\prime \prime}+\frac{b}{t} \zeta^{\prime}=c a^{p+2} t^{p} \zeta^{q} \text { for } 0<t<1, \\
\zeta(0)=1, \zeta(1)=0 .
\end{gathered}
$$

From Theorem 1 or 2 , the problem (3.8) has a unique solution $\zeta$, which is positive for $0 \leqslant t<1$.

The following result generalizes the first part of Theorem 5.2 of Mooney. Its proof is different from his.

THEOREM 3. If $0<r<s$, and $\zeta_{r}(t)$ and $\zeta_{s}(t)$ are solutions of the problem (3.8) with $a$ equal to $r$ and $s$, respectively, then $\zeta_{r}(t)>\zeta_{s}(t)$ for $0<t<1$.

Proof. From (3.8),

$$
\begin{gathered}
\left(\zeta_{r}-\zeta_{s}\right)^{\prime \prime}+\frac{b}{t}\left(\zeta_{r}-\zeta_{s}\right)^{\prime}=c t^{p}\left(r^{p+2} \zeta_{r}^{q}-s^{p+2 \zeta_{s}^{q}}\right), \\
\zeta_{r}(0)-\zeta_{s}(0)=0=\zeta_{r}(1)-\zeta_{s}(1) .
\end{gathered}
$$

If $\zeta_{r} \leqslant \zeta_{s}$ somewhere in the interval $(0,1)$, then from (3.9), and the positivity of $\zeta_{r}$ and $\zeta_{s}$, there exists a subinterval $\left(t_{1}, t_{2}\right)$ such that the right-hand side of (3.9) is nonpositive, and $\zeta_{r}-\zeta_{s}$ attains its nonpositive minimum $m$ somewhere there. By the maximum principle, $\zeta_{r}-\zeta_{s} \equiv m$ for $t_{1}<t<t_{2}$. It follows that $t_{1}=0, t_{2}=1$, and $m=0$ by continuity. This contradicts the fact that the solutions $\zeta_{r}$ and $\zeta_{s}$ are unique. Thus, the theorem is proved.

4. Numerical solutions. In this section, we apply our constructive method to obtain lower bounds, upper bounds, and the solution of the problem (1.5) and (1.4). Each successive approximation is given explicitly by an analytic solution.

With $y_{1}=1-x / a-U_{1}$, it follows from (3.4) that

$$
L y_{1}=0, \quad y_{1}(0)=1, \quad y_{1}(a)=0 .
$$

From (3.5) and (3.6), we obtain

$$
y_{1}(x)=\frac{2}{\Gamma(\nu)}\left|\frac{\alpha}{2}\right|^{\nu}\left[w_{2}(x)-w_{1}(x) / k\right]
$$

which gives a lower bound of the problem (1.5) and (1.4). From (3.4) and (2.5),

$$
U_{n+1}(x)=-\int_{0}^{a} G(x ; \xi) \xi^{p+h}\left\{c\left[1-\frac{\xi}{a}-U_{n}(\xi)\right]^{q}+c q U_{n}(\xi)+\frac{b}{a} \xi^{-(p+1)}\right\} d \xi
$$

for $n=1,2,3, \cdots$. Hence, lower bounds of the solution are given by

$$
\begin{aligned}
y_{n+1}(x)=1-\frac{x}{a}+\int_{0}^{a} G(x ; \xi) \xi^{p+h}\left\{c\left[1-\frac{\xi}{a}-U_{n}(\xi)\right]^{q}\right. & \\
& \left.+c q U_{n}(\xi)+\frac{b}{a} \xi^{-(p+1)}\right\} d \xi,
\end{aligned}
$$

where $y_{n+1}=1-x / a-U_{n+1}$. 
For $n=0,1,2, \cdots$, let $Y_{n}=1-x / a-u_{n}$. It follows from (3.7) and (2.5) that the upper bounds of the solution are given by

$$
\begin{aligned}
Y_{n+1}(x)=1-\frac{x}{a}+\int_{0}^{a} G(x ; \xi) \xi^{p+b}\left\{c\left[1-\frac{\xi}{a}-u_{n}(\xi)\right]^{q}\right. & \\
& \left.+c q u_{n}(\xi)+\frac{b}{a} \xi^{-(p+1)}\right\} d \xi .
\end{aligned}
$$

The calculations for the following two examples (with $a=1, c=1, p=-\frac{1}{2}$, and $q$ $\left.=\frac{3}{2}\right)$ are done on Honeywell $68 / 80$ Multics computer system. We divide the interval $[0,1]$ into ten equal subintervals.

In our first example, we let $b=\frac{1}{4}$. This yields $\nu=\frac{1}{2},|\alpha|=\left(\frac{8}{3}\right)^{1 / 2}$, and $\beta=\frac{3}{4}$. To compute the second lower bound $y_{1}$ numerically from (4.1), we use subroutines from the IMSL Library: MGAMAD (= DGAMMA to evaluate the gamma function of a double precision argument), MMBSIR (to compute, to double precision, a modified Bessel function of the first kind of nonnegative real order for real positive arguments with exponential scaling option), and MMBSKR (to compute, to double precision, a modified Bessel function of the second kind of nonnegative real fractional order for real positive arguments scaled by exp(arg)). To obtain the second upper bound $Y_{1}$ numerically from (4.3), we use subroutines MGAMAD, MMBSIR, MMBSKR, and DCADRE (for numerical integration, to single precision, of a function using cautious adaptive Romberg extrapolation). To compute $y_{n+1}$ and $Y_{n+1}$, respectively, from (4.2) and (4.3) for $n=$ $1,2,3, \cdots$, we use subroutines ICSCCU (to perform, to single precision, a cubic spline interpolation), MMBSIR, MMBSKR, ICSEVU (to evaluate, to single precision, a cubic spline) and DCADRE. We find that $y_{6}(x)=Y_{6}(x)$ to five decimal digits. Hence, either one of these rounded to five decimal digits can be taken to be the solution $y$. The results for $y_{1}, Y_{1}$, and $y$ are given in Table 1 .

TABLE 1

for $a=1, b=\frac{1}{4}, c=1, p=-\frac{1}{2}, q=\frac{3}{2}$

$\begin{array}{cccc}x & y_{1} & Y_{1} & y \\ 0.00000 & 1.00000 & 1.00000 & 1.00000 \\ 0.10000 & 0.72460 & 0.77089 & 0.76615 \\ 0.20000 & 0.57331 & 0.63714 & 0.62946 \\ 0.30000 & 0.45940 & 0.53023 & 0.52056 \\ 0.40000 & 0.36709 & 0.43799 & 0.42727 \\ 0.50000 & 0.28898 & 0.35487 & 0.34405 \\ 0.60000 & 0.22074 & 0.27776 & 0.26775 \\ 0.70000 & 0.15953 & 0.20471 & 0.19635 \\ 0.80000 & 0.10330 & 0.13452 & 0.12850 \\ 0.90000 & 0.05053 & 0.06641 & 0.06326 \\ 1.00000 & 0.00000 & 0.00000 & 0.00000\end{array}$


In our second example, we let $b=0$. This is the case of an ionized atom described by the Thomas-Fermi equation (1.1) subject to the boundary conditions (1.4). Here $\nu=\frac{2}{3}$, $|\alpha|=\left(\frac{8}{3}\right)^{1 / 2}$, and $\beta=\frac{3}{4}$. We use the same IMSL subroutines as in the last example to do the numerical computations. We compute the lower bound $y_{1}$ and the upper bound $Y_{1}$ from (4.1) and (4.3), respectively. Their values (given in Table 2) are the same as Mooney's results (cf. his Table 3 ), which are obtained in the form of power series expanded about the origin. Using (4.2) and (4.3), we find that $y_{5}(x)=Y_{5}(x)$ to five decimal digits. Again, either one of these rounded to five decimal digits gives the solution $y$. The results are given in Table 2.

\section{TABLE 2}

\begin{tabular}{cccc}
\multicolumn{4}{c}{ for $a=1, b=0, c=1, p=-\frac{1}{2}, q=\frac{3}{2}$} \\
$x$ & $y_{1}$ & $Y_{1}$ & $y$ \\
\hdashline 0.00000 & 1.00000 & 1.00000 & 1.00000 \\
0.10000 & 0.81634 & 0.85072 & 0.84947 \\
0.20000 & 0.67664 & 0.72962 & 0.72723 \\
0.30000 & 0.56026 & 0.62261 & 0.61929 \\
0.40000 & 0.45947 & 0.52433 & 0.52041 \\
0.50000 & 0.36968 & 0.43169 & 0.42755 \\
0.60000 & 0.28780 & 0.34265 & 0.33869 \\
0.70000 & 0.21154 & 0.25580 & 0.25240 \\
0.80000 & 0.13909 & 0.17014 & 0.16765 \\
0.90000 & 0.06899 & 0.08501 & 0.08369 \\
1.00000 & 0.00000 & 0.00000 & 0.00000
\end{tabular}

\section{REFERENCES}

[1] N. Anderson and A. M. Arthurs, Variationai solutions of the Thomas-Fermi equation, Quart. Appl. Math. 39. $127-129$ (1981)

[2] B. L. Burrows and P. W. Core, A variational-iterative approximate solution of the Thomas-Fermi equation, Quart. Appl. Math. 42, 73-76 (1984)

[3] V. Bush and S. H. Caldwell, Thomas-Fermi equation solution by the differential analyzer, Phys. Rev. 38. $1898-1901(1931)$

[4] C. Y. Chan and S. W. Du, A constructive method for the Thomas-Fermi equation, Quart. Appl. Math. 44, 303-307 (1986)

[5] E. Fermi, Un metodo statistico per la determinazione di alcune proprietá dell' atomo, Rend. Accad. Naz. del Lincei, Cl. Sci. Fis., Mat. e. Nat. (6) 6, 602-607 (1927)

[6] H. B. Keller and D. S. Cohen, Some positone problems suggested by nonlinear heat generation, J. Math. Mech. 16, 1361-1376 (1967)

[7] C. D. Luning, An iterative technique for obtaining solutions of a Thomas-Fermi equation, SIAM J. Math. Anal. 9. 515-523 (1978)

[8] J. W. Mooney, Monotone methods for the Thomas-Fermi equation, Quart. Appl. Math. 36, 305-314 (1978)

[9] M. H. Protter and H. F. Weinberger, Maximum principles in differential equations, Prentice-Hall, Inc., Englewood Cliffs, New Jersey, 1967, p. 6 
[10] R. V. Ramnath, A new analytic approximation for the Thomas-Fermi model in atomic physics, J. Math. Anal. Appl. 31, 285-296 (1970)

[11] H. L. Royden, Real analysis, 2nd ed., Macmillan Publishing Co., New York, New York, 1968, p. 88

[12] A. Sommerfeld, Asymptotische integration der Differential-Gleichung des Thomas-Fermischen Atoms, Z. Phys. 78, 283-308 (1932)

[13] L. H. Thomas, The calculation of atomic fields, Proc. Cambridge Philos. Soc. 23, 542-548 (1927)

[14] G. N. Watson, A treatise on the theory of Bessel functions, 2nd ed., Macmillan Co., New York, New York, 1944, pp. 80,97 